\title{
Golpe de estado en la biosfera: los ecosistemas al servicio del capital
}

\author{
Yayo HERRERO \\ Ecologistas en Accións
}

Recibido: 12.12 .2011

Aceptado: 25.01.2012

\section{RESUMEN}

Las sociedades capitalistas se han construido de espaldas a las bases materiales que sostienen la vida. La obsesión por el crecimiento económico y la acumulación ha declarado la guerra a los cuerpos y a los territorios. La vida humana, como el resto de lo vivo, depende de la biosfera, de sus bienes y sus procesos, y también de la cantidad enorme de trabajo y energía que supone ocuparse de los cuerpos vulnerables. Las sinergias entre dos visiones heterodoxas de la economía, la economía ecológica y la economía feminista, contribuyen a alumbrar otro paradigma que sitúe el bienestar para todas las personas y la conservación de la vida humana y natural en el centro del interés.

Palabras clave: Límites, ciclos, solar, huella y deuda ecológica, huella y deuda de cuidados.

\section{Coup d'etat in the biosphere: the ecosystem at the capital service}

\begin{abstract}
Capitalist societies have been established denying the material basis that sustains life. The obsession with the idea of economic growth and accumulation has declared the war to bodies and territories. Human life, as well as all which is alive, depends on the biosphere, its assets and processes, and on the enormous workload that taking care of vulnerable bodies implies. Synergies between two heterodox economic approaches, ecological economics and feminist economics, contribute to the constitution of a paradigm focused on universal wellbeing and on the maintenance of human and natural life.
\end{abstract}

Key words: limits, cycles, ecological footprint and ecological debt, care footprint and care debt.

Hace varias décadas el informe Meadows (Meadows, 1972), publicado por el Club de Roma, constataba la evidente inviabilidad del crecimiento permanente de la población y sus consumos. Alertaba de que en un mundo físicamente limitado, el crecimiento continuo de la extracción de materiales, de la contaminación de aguas, tierra y aire, de la degradación de los ecosistemas, simplemente no era posible. Aquel informe advertía con preocupación que, de no revertirse la tendencia al crecimiento exponencial de todos esos factores, se incurriría en el riesgo de llegar a superar los límites del planeta, ya que el crecimiento continuado y exponencial sólo podía darse en el mundo físico transitoriamente.

Más de 30 años después, una repetición del mismo ejercicio mostraba que, lejos de encararse el problema, se había profundizado esta dinámica (Meadows et al 
.2004). La humanidad ya no se encuentra en riesgo de superar los límites de la biosfera, sino que ha llegado a una situación de translimitación (García., 2005:115).

El crecimiento económico como única prioridad de la sociedad capitalista ha conducido en apenas un par de siglos a superar los límites biogeofísicos del planeta. Los sumideros que deben degradar los residuos que genera el proceso económico no dan a basto y los servicios que prestan los ecosistemas comienzan a dar muestras de deterioro y cambio.

Pero el modelo socioeconómico capitalista no se ha expandido sólo a costa de los sistemas naturales, sino también a partir de la incautación de los tiempos de las personas para ponerlos al servicio del mercado. Es evidente en el caso de las personas empleadas en el mercado laboral en el que venden su fuerza de trabajo a cambio de un salario. Sin embargo, la apropiación ha sido menos visible o totalmente invisible en lo referente a los tiempos dedicados a la reproducción social y mantenimiento de la vida cotidiana. El cuidado de los cuerpos vulnerables constituye un elemento profundamente material e insoslayable para la supervivencia humana, pero debido a la lógica patriarcal esta tarea exigente, imprescindible y muchas veces penosa recae de forma casi exclusiva en las mujeres.

La desvinculación entre la economía capitalista y las bases materiales que permiten la vida, la ignorancia de la dependencia radical que tenemos los seres humanos de los ciclos y bienes naturales y del cuidados de los cuerpos, ha conducido a una forma de entender la sociedad, la economía o la cultura que está transformando profundamente muchos de los elementos que sustentan la condición humana: impide el acceso equitativo a los recursos y bienes que proporciona el planeta Tierra; dificulta la posibilidad de los hogares sigan manteniendo la reproducción social y perpetúa la lógica patriarcal de división del trabajo que obliga a las mujeres a ser las únicas responsables de mantener el bienestar cotidiano; destruye las dinámicas cooperativas que hicieron del "animal" humano ante todo un ser social; anula la viabilidad de las generaciones futuras sobre la Tierra; simplifica los procesos de interdependencia que nos han hecho coevolucionar con muchas otras especies compañeras de aventura planetaria...

El conocimiento procedente de múltiples ámbitos del saber (ecología, sociología, economía, los feminismos, la filosofía, geografía, historia) viene planteando desde hace décadas los riesgos, potencialmente catastróficos en los que estamos incurriendo, señalando que la actual crisis no es sólo económica, financiera, social o ecológica. Todas ellas operan de forma sinérgica e interaccionan unas con otras. No es posible afrontar una sola de estas dimensiones sin operar sobre las otras. Nos encontramos por tanto ante una crisis civilizatoria, que nos obliga a repensar y reconducir nuestro actual modelo hacia otro que pueda ser viable y justo. 
Pero además, el cambio no puede ser lento, por más que sea difícil. Algunos de los principales problemas ecológicos que afrontamos tendrán que ser encarados en las próximas décadas. El cambio climático o el agotamiento del combustible fósil nos obligan a emprender profundos cambios estructurales en apenas diez o quince años.

\section{LA CRISIS ECOLÓGICA}

Naredo pone de manifiesto cómo hasta la llegada de la Revolución Industrial, los hombres y las mujeres, al igual que el resto del mundo vivo, vivieron de los recursos que proporcionaba la fotosíntesis y de los materiales que encontraban en su entorno más próximo (Naredo, 2006: 47).

Los seres humanos aseguraban su supervivencia imitando el funcionamiento de la biosfera. La economía se basaba en el mantenimiento de la diversidad que existía. Todo era objeto de un uso posterior, en una cadena, un ciclo, que aseguraba la renovación de los materiales empleados. Los ritmos de vida eran los marcados por los ciclos de la naturaleza y éstos eran dinamizados por la energía del sol.

Sin embargo, las sociedades se alejaron del funcionamiento de la biosfera al comenzar a utilizar la energía de origen fósil para acelerar las extracciones y las producciones. La disponibilidad, primero de carbón, y luego de gas natural y petróleo, posibilitó un cambio profundo en el metabolismo económico y la posibilidad de superar los límites del territorio en el que se vivía mediante un sistema de transporte que permitía obtener energía, materiales y alimentación procedente de territorios lejanos.

Este crecimiento masivo, sin consideración de límites, apoyado en el manejo a gran escala de los stocks de los materiales contenidos en la corteza terrestre, conduce al deterioro del patrimonio natural que ha legado la evolución, tanto por la extracción de recursos no renovables, como por la generación de residuos, resultando en el extremo globalmente inviable.

Durante los siglos XIX y XX se pensaba que la biosfera era un espacio inagotable, pero bruscamente hemos superado ya su biocapacidad. Los límites biofísicos y las contradicciones internas del propio proceso de funcionamiento económico son ya insoslayables. Tal y como señala Fernández Durán,

"la guerra silenciosa, destructiva y en acelerado ascenso contra la Naturaleza llevada a cabo por la expansión a escala planetaria del sistema urbano-agro-industrial ya no se puede ocultar, y está actuando actualmente como un auténtico boomerang contra el mismo" (Fernández Durán, 2010:5).

Hablamos de la modificación del funcionamiento del clima de la Tierra, de la composición y características de sus sistemas hidrológicos, de la magnitud, diversi- 
dad y complejidad de la biodiversidad planetaria, de la transformación del propio paisaje y territorio.

La actual crisis ecológica se refleja en una gran cantidad de fenómenos interrelacionados que amenazan con transformar las condiciones biofísicas a las cuales la especie humana está adaptada. En la base de todas esas manifestaciones de la crisis ecológica se encuentra un elemento común: la incompatibilidad esencial que existe entre un planeta físicamente limitado y una forma de organización socioeconómica basada en la expansión continuada de la producción y el consumo.

\subsection{NADA PUEDE CRECER INDEFINIDAMENTE EN UN PLANETA CON LÍMITES}

El planeta Tierra cuenta con una cantidad finita de materiales y por tanto la extracción y uso de los mismos no puede ser ilimitada. Los sumideros que degradan los desechos y residuos que genera cualquier actividad, también presentan límites. Llamamos recursos naturales a los bienes, servicios o funciones útiles del medioambiente biofísico que satisfacen necesidades humanas (o más bien en muchos casos, deseos). Son recursos tanto las fuentes de energía libre y materiales ordenados, como los sumideros (vertederos) de energía disipada y materiales degradados (García 2004).

Los recursos no renovables (o renovables sólo en tiempo geológicos) están limitados por la cantidad total disponible. Los renovables no están limitados en cantidad si se respetan sus ritmos de regeneración. La energía solar, por ejemplo, no está limitada por la cantidad total ni por la tasa de uso, pero sí lo está por el hecho de que la estructura de captación (los seres que realizan la fotosíntesis o las placas solares) es finita.

Si el planeta está sujeto a límites, tanto desde el punto de vista de las fuentes de recursos como de las posibilidades de degradar residuos, dentro de él nada puede crecer indefinidamente, ya sea una persona, un encinar, o un arrecife coralino. El uso presente de recursos no renovables (petróleo, carbón o minerales) es lesivo para las generaciones futuras y refleja la práctica absurda de celebrar como riqueza la desaparición irreversible del patrimonio natural.

El ineludible hecho de que el sistema económico se encuentre dentro del sistema de la biosfera, de que requiera materiales y energía, así como emitir residuos y calor, implica que no pueda plantearse en términos de crecimiento ilimitado. Ignorar la dinámica de la naturaleza ha desencadenado un golpe de estado biológico que ha puesto a trabajar los ciclos naturales al servicio de la obtención de beneficios provocando una serie de deterioros que colocan el futuro de la especie humana en una situación muy comprometida. 


\subsection{EL CAMBIO CLIMÁTICO}

Se utiliza el término efecto invernadero para señalar la importancia de la atmósfera de cara a calentar la superficie de la tierra. La atmósfera es casi transparente a la luz que llega del sol (la luz visible e infrarroja de onda corta). La mayor parte de ella es absorbida y posteriormente devuelta a la atmósfera, en donde una parte se transforma en calor al ser captada por algunos gases que se encuentran presentes en ella. La atmósfera, gracias a estos gases, recupera parte de la energía del sol que pretende escapar, impidiendo que la tierra se enfríe.

Actualmente, las concentraciones de los gases de efecto invernadero se han disparado debido, fundamentalmente, a la combustión de energías fósiles y a los cambios de uso del suelo. La cantidad de calor que retiene la atmósfera es mucho mayor y en consecuencia la temperatura global terrestre está aumentando. Este calentamiento está desencadenando un cambio climático que se traduce en una alteración global de los regímenes de precipitaciones (cantidad de lluvias, distribución, fenómenos catastróficos), de las dinámicas de las aguas marinas (nivel, temperatura, corrientes), de las interacciones que se dan en los ecosistemas, además de una diferente distribución de tierras y mares por el ascenso del nivel del mar (Moreno, 2005, Duarte, 2006).

La subida rápida de la temperatura media del planeta influye en los ciclos de vida de muchos animales y plantas, que, sin tiempo para la readaptación, serán incapaces de alimentarse o de reproducirse. También supone la reaparición de enfermedades ya erradicadas de determinadas latitudes. La alteración del régimen de lluvias implica sequías y lluvias torrenciales que dificultan gravemente la supervivencia de las poblaciones que practican la agricultura y ganadería de subsistencia. El deshielo de los polos derivará en la inundación progresiva de las costas y la pérdida de hábitat de sus pobladores. La reducción de las poblaciones de determinadas especies animales y vegetales repercute en la supervivencia de otras especies dependientes de estas, y la cadena de interdependencias arrastra a todo su ecosistema. Estos cambios dificultan la producción de alimentos para los seres humanos.

En los estudios proyectivos del Grupo Intergubernamental de Expertos sobre el Cambio Climático (IPCC) se augura que, de superarse el umbral de los dos grados de subida media, las alteraciones de las condiciones ambientales serán tales que puede llegarse a un punto sin retorno en el que se desencadenen fenómenos imprevisibles y catastróficos.

De no reducir de una forma significativa las emisiones de gases de efecto invernadero la situación puede ser dramática. Pero una reducción significativa de emisiones en los países más ricos, que son los que más emiten y mayor responsabilidad histórica tienen, significa un cambio importante en los modos de producción, las tasas de ganancia, el consumo, el comercio y la movilidad en estos países. No es de extrañar que al mismo tiempo que se escriben estas líneas y mientras la 
Unión Europea aprueba drásticos recortes sociales para transferir riqueza de las personas a los capitalistas, en la Cumbre del Clima de Durban, los países más contaminantes se nieguen a reducir sus emisiones, aunque eso ponga en una situación tremendamente vulnerable a muchas personas en los países de la Periferia.

Según el PNUD (2008), los fenómenos naturales golpearán con mayor fuerza a las regiones y a las personas más pobres. Las mujeres pobres serán las más afectadas por el cambio climático, ya que son ellas las encargadas de administrar los alimentos, el combustible y, en algunas regiones, el forraje y agua. Los efectos negativos del cambio climático son más intensos en las comunidades con mayor pobreza, donde la mayor parte de la carga social recae en las mujeres.

La mayor vulnerabilidad de las mujeres frente al cambio climático no es una condición natural, es un resultado del funcionamiento de dinámicas sociales, económicas y culturales. Es posible acometer acciones específicas para transformar esta situación y dar un mayor protagonismo de las mujeres en los planes de acción de mitigación y adaptación al cambio climático de los estados, municipios y localidades.

\subsection{EL AGOTAMIENTO DE LOS RECURSOS NATURALES}

Nos encontramos ante lo que hace años Hubbert denominó el "pico del petróleo" (Hubbert, 1949), es decir el momento en el se han extraído la mitad de las reservas existente y por tanto se ha alcanzado un máximo de extracción. La propia Agencia Internacional de la Energía ha manifestado que el pico del petróleo se alcanzó en 2006. Una vez alcanzado este pico, la obtención de petróleo comenzaría a declinar. Cada vez se va agrandando más la brecha entre una demanda creciente y unas reservas que se agotan y cuya dificultad y coste de extracción aumenta.

Muchos de los yacimientos actuales obligan a hacer prospecciones más profundas, a crear plataformas en medio del mar o a procesos de depuración muy costosos y arriesgados ${ }^{1}$. Ante este horizonte de declive incluso las empresas petroleras empiezan a sopesar y poner en marcha fuentes de energía alternativas que permitan mantener el creciente consumo de energía, recurriendo por ejemplo a la energía solar, la eólica o a la biomasa. Sin embargo, ninguna de ellas tiene poder energético de las energías fósiles. Sus tasas de retorno (la relación entre la energía que se invierte para producirla y la energía finalmente producida) son mucho menores (Ballenilla, 2007). Eso sin contar con el sustrato físico de materiales, también finitos, necesario para fabricar los aparatos que permiten la captación y acumulación de energía.

1 Estos procesos industriales de riesgo pueden dar lugar a catástrofes ecológicas como el vertido de crudo en el Golfo de México causad por la petrolera BP. 
Las energías renovables y limpias pueden satisfacer las necesidades humanas pero no a la escala de las exigencias de un modelo de producción, distribución consumo, sumamente energívoro, que además pretende continuar creciendo (Fernández Durán, 2008).

La economía capitalista ha crecido a expensas de la energía barata y aparentemente inagotable que proporcionaba el petróleo (Naredo, 2006: 47). Éste ha servido para mover máquinas e impulsar vehículos de automoción, para producir electricidad. Ha permitido que las personas puedan trabajar a decenas de kilómetros de su lugar de residencia y que se alimenten a diario con productos baratos cultivados e territorios lejanos. El petróleo es imprescindible en la agricultura intensiva y en la producción de insumos agrícolas, lo es también en la fabricación de ropas, casas, muebles, carreteras, envases... Las grandes urbes son inviables sin energía abundante y barata. Vivimos en un mundo construido con petróleo y su agotamiento, queramos o no modificará todo el modelo de vida.

No sólo se trata de la energía fósil, la velocidad a la que se están consumiendo también otros recursos naturales es incompatible con los ritmos que requiere la naturaleza para regenerarlos, por lo que ya ha comenzado a manifestarse la progresiva escasez de otros recursos imprescindibles para la vida como son el agua dulce, los bosques, la pesca, los suelos fértiles, la fauna salvaje o los arrecifes de coral.

\subsection{LA PÉRDIDA DE BIODIVERSIDAD}

La pérdida de biodiversidad es el despilfarro de nuestro mayor seguro de vida. Nos encontramos ante la sexta gran extinción masiva, y la primera provocada por una especie, la humana (Oberhuber, 2004). Esta disminución de la biodiversidad se acompaña también de una pérdida de diversidad cultural. La imposición de los modelos de vida occidentales aniquila rápidamente la enorme variedad de comportamientos culturales que la humanidad creó a lo largo de su evolución. El conocimiento que permite producir alimentos cuando el agua es escasa, o construir casas que no requieran combustible fósil para mantenerse a una temperatura adecuada, desaparece rápidamente, sin que las personas seamos conscientes de la gravedad de esta pérdida. Muchas poblaciones de plantas y animales que todavía subsisten han disminuido su número y extensión, lo que las coloca al borde de la desaparición. El ritmo de extinción de especies está siendo 100 veces más rápido que su velocidad natural y cada vez se intensifica más.

La biodiversidad está en la misma base de la vida en la Tierra y es el principal sustento de nuestra existencia. Esta dependencia permanece oculta e invisible a la lógica económica. No hay reemplazo posible y a nuestro alcance para reconstruir artificialmente la biodiversidad. Su pérdida está afectando ya a ciclos vitales como el del agua o el del carbono. El panorama de deterioro global se completa si añadimos el aumento de incertidumbre que suponen la proliferación de la industria 
nuclear, la comercialización de miles de nuevos productos químicos al entorno que interfieren con los intercambios químicos que regulan los sistemas vivos, la liberación de organismos genéticamente modificados cuyos efectos son imprevisibles o la experimentación en biotecnología y nanotecnología cuyas consecuencias se desconocen.

\section{LA CRISIS SOCIAL}

La crisis ecológica también tiene su expresión en el ámbito social. El sistema económico basado en el crecimiento continuado se ha mostrado incapaz de satisfacer las necesidades vitales de la mayoría de la población. Hasta el presente los sectores sociales con más poder y más favorecidos han podido superar los límites de sus propios territorios recurriendo a la importación de biodiversidad y "servicios ambientales" de otras zonas del mundo poco degradadas y con abundancia de recursos. Pero esto está dejando de ser así, y estas áreas también se comienzan a deteriorar, agravando la situación de las poblaciones más empobrecidas del mundo que llevan ya décadas sufriendo esta guerra ambiental encubierta.

Son muy conocidos los datos que muestran las enormes desigualdades sociales entre el Centro y la Periferia en términos de renta. Pero las diferencias en términos físicos son también enormes. La sexta parte de la población mundial, principalmente ubicada en los países enriquecidos consume el $80 \%$ de los recursos disponibles, mientras que los 5/6 restantes utilizan el 20\% restante de los recursos. Según el informe Planeta Vivo (WWF, 2010: 38-39), se calcula que a cada persona le corresponden alrededor 1,8 hectáreas globales de terrenos productivos por persona. Pues bien, la media de consumo mundial supera las 2,2has y este consumo no es homogéneo. Mientras que en muchos países del Sur no se llega a las 0,9, la ciudadanía de Estados Unidos consume en promedio 8,2 hectáreas per cápita, la canadiense 6,5, y la española unas 5.5 Has. Si toda la población del planeta utilizase los recursos naturales y los sumideros de residuos como la media de una persona española, harían falta más de tres planetas para poder sostener ese estilo de vida. Es la tónica de cualquier país desarrollado y pone de manifiesto la inviabilidad física de extender este modelo a todo el mundo.

El deterioro de los territorios que han habitado una buena parte de los pueblos del Sur durante miles de años, y de sus condiciones básicas de existencia, ha expulsado a las personas, obligando a unos movimientos migratorios sin precedentes. Muchos pueblos han sido desposeídos de su derecho a permanecer y se ven obligados a seguir la misma ruta que siguen las materias primas y los frutos de los monocultivos que se extraen de los lugares donde antes vivían: el viaje del Sur al Norte. Además, las desigualdades dentro del propio Norte y el Sur son también relevantes. Las crisis y los planes de ajuste del FMI aplicados en Grecia, Italia, Portugal o España están creando enormes bolsas de pobreza, de exclusión y de privación de bienes básicos como la vivienda o la salud. 
El deterioro ambiental impacta de lleno en las comunidades humanas y sus modos de vida. Martínez Alier muestra cómo en todos los lugares del mundo la irracional y creciente explotación de los recursos naturales no sólo da origen a problemas ambientales, sino también a numerosos y gravísimos conflictos sociales. Algunos de los conflictos ecológicos-distributivos son (Martínez Alier, 2004):

-Conflictos mineros evidenciados por las quejas sobre minas y fundiciones a causa de la contaminación del suelo, del aire y del agua, y por la ocupación de tierras por la minería a cielo abierto y las escorias.

-Conflictos por la extracción de petróleo a causa de la contaminación del aire, del suelo y de las aguas. Degradación y erosión de las tierras, causadas por la desigual distribución de la propiedad sobre la tierra, por la presión de la producción exportadora y los monocultivos.

-Sustitución de los bosques por plantaciones de árboles destinados a la industria del papel. Destrucción de los manglares y de las poblaciones locales cuya subsistencia depende de ellos por la industria camaronera de exportación.

-Biopiratería, apropiación y mercantilización de los recursos biológicos, tanto "silvestres" como medicinales y agrícolas, sin reconocimiento del conocimiento y propiedad de los indígenas y campesinos sobre ellos y sin compensación alguna.

-Conflictos sobre el agua, movimientos contra las grandes represas para hidroelectricidad e irrigación y problemas. También los conflictos por el uso y contaminación de acuíferos y los conflictos por trasvases de ríos.

-Conflictos por los intentos de evitar la sobrepesca industrial en detrimento de la pesca artesanal.

-Conflictos sobre el transporte que nacen del trasiego cada vez mayor de materiales y energía: derrames petroleros en el mar, guerras relacionadas con oleoductos o gasoductos, ampliaciones de puertos y aeropuertos, construcción de nuevas autopistas.

-Luchas tóxicas ante los riesgos que causan los metales pesados, dioxinas, etc. -Contaminación transfronteriza: emisiones de dióxido de azufre que cruzaban fronteras y producían lluvia ácida, contaminaciones radioactivas por ensayos de armas nucleares en el Pacífico, por ejemplo emisiones de CFC que han dañado la capa de ozono.

-Conflictos por los derechos iguales a los sumideros de carbono.

Los impactos físicos y sociales de estos conflictos han conducido a acuñar el concepto de deuda ecológica (Martínez Alier, 2004) para reflejar la desigual apropiación de recursos naturales, territorio y sumideros por parte de los países enriquecidos. Estos países habrían contraído una deuda física con los países empobrecidos al superar las capacidades de sus propios territorios y utilizar el resto del mundo como mina y vertedero. 


\subsection{LA SOCIEDAD DEL CRECIMIENTO CREA UN BIENESTAR ILUSORIO}

Una buena parte del bienestar que crea un modelo económico que ignora las dinámicas naturales y la equidad entre personas es engañoso. El progreso y el éxito económico en nuestro sistema cultural se suele medir por la cantidad de actividad económica en el mercado que tiene un país, ignorando los costes físicos y sociales reales de la producción y de la reproducción. Concebido de esta forma, crecimiento económico se equipara a bienestar y calidad de vida y se mide a través del indicador por excelencia de la riqueza, el Producto Interior Bruto (PIB), la fórmula más reconocida para evaluar el comportamiento económico, que se obtiene sumando simplemente agregados monetarios.

Esta forma de contabilizar la riqueza hace que se sumen en el lado positivo, y que cuenten como riqueza, cualquier producción y gasto, incluso los que son perjudiciales y los que se producen para paliar el deterioro. A la vez, se ocultan muchas producciones valiosas pero no monetizadas, al mismo tiempo que no resta lo que se destruye. Las guerras, las enfermedades y el gasto farmacéutico, el incremento de tráfico motorizado o la construcción de infraestructuras, suman en el PIB, mientras que la destrucción irreversible asociada a estos procesos no resta en ningún sitio. Sin embargo, el aire limpio, los trabajos relacionados con los cuidados de la vida humana y la reproducción social, la propia renovación generacional de la mano de obra, el trabajo de la fotosíntesis que realizan las plantas, o los servicios del regulación del clima que realiza la Naturaleza, siendo imprescindibles para el mantenimiento la vida, no suman en ningún lugar.

Se podría esperar que esa sexta parte de la población mundial que vive en las zonas favorecidas del planeta a costa de los recursos de territorios lejanos disfrutara de la máxima calidad de vida. Sin embargo, después de algunas décadas de fuerte consumo de energía y materiales se observan numerosos problemas": impermeabilización del territorio, contaminación en las ciudades, incremento de las enfermedades depresivas, estrés y ansiedad, fuerte simplificación de los ecosistemas, falta de seguridad alimentaria, dificultad de acceso a la vivienda, etc.

La creación de riqueza para las personas durante los períodos de crecimiento económico también tiene mucho de espejismo. Durante el periodo de mayor crecimiento económico del estado español, entre 1994 y 2007, mientras las personas tenían la percepción subjetiva de que prosperaban y aumentaba la riqueza, en realidad los salarios medios bajaban y el acceso a más bienes y servicios de consumo se producía a partir de endeudamiento de personas y empresas. Durante ese periodo de crecimiento, una ola de cemento sepultó buena parte del litoral dejando las costas plagadas de casas adosadas que tienen un nivel de ocupación de 22 días al año.

Una vez que la burbuja estalla, las reacciones de los gobiernos al servicio de los grandes capitales es favorecer el expolio de lo poco público que queda. Se recortan servicios públicos básicos para el bienestar de las personas y se transfieren esos 
servicios, que no se pueden dejar de hacer, al entorno de los hogares y, dadas las relaciones de poder que se dan en las familias, es muy probable que la mayor parte de la tensión y del ajuste caiga mayoritariamente sobre las mujeres.

La actual crisis civilizatoria es también una crisis de la forma en que se percibe y valora la riqueza. Uno de los ejes centrales de cambio avanzar hacia una cultura que asuma que vive en un mundo con límites. Límites en cuanto a la naturaleza y en cuanto al propio cuerpo humano vulnerable y finito. Aceptar la existencia de ambos límites es imprescindible para la consecución de una vida digna para todas las personas compatible con la sostenibilidad del planeta. Ambos extremos son imposibles si una parte de la humanidad acumula riquezas de forma injusta y el resto sueña ilusoriamente poder ser como ellos.

\subsection{LA CRISIS DE CUIDADOS EN NUESTRAS SOCIEDADES URBANAS}

Vamos a considerar en este epígrafe sólo algunos aspectos de la crisis de cuidados que se relacionan especialmente con la crisis ecológica. La crisis de cuidados será tratada de una forma más amplia en otros artículos de la revista.

En las últimas décadas se han dado una serie de circunstancias que han alterado profundamente el modelo previo de reparto de las tareas domésticas y de cuidados que configura la base sobre la que se sostienen las estructuras económicas, el mercado laboral y el mantenimiento de la vida humana. La crisis de los cuidados es el resultado de la confluencia de un conjunto de factores entre los que destaca el acceso de las mujeres al empleo remunerado dentro de un sistema patriarcal. La posibilidad de que las mujeres sean sujetos políticos de derecho se materializa como algo vinculado a la consecución de independencia económica a través del empleo. Pero el trabajo doméstico no puede dejar de hacerse y el paso de las mujeres al mundo público del empleo no se ha visto acompañado por un reparto de los trabajos de cuidados con los hombres, y la sociedad y el estado tampoco se responsabilizan de las tareas de reproducción social.

Dado que hay que seguir atendiendo a las personas ancianas, a la infancia y a las personas con diversidad funcional, que la mayor parte de los hombres no se hacen responsables de estas tareas y que los servicios públicos no cubren las necesidades reales, las mujeres acaban asumiendo dobles o triples jornadas y transfiriendo parte de esos trabajos a otras mujeres de la familia, o, cuando las relaciones de clase lo permiten, a mujeres contratadas que realizan estos trabajos en condiciones frecuentemente precarias.

Es especialmente notorio el papel que juegan las mujeres migrantes en los trabajos de cuidados. Se crea una cadena global de cuidados en la que las mujeres migrantes que asumen como empleo precario el cuidado de la infancia, de las personas mayores y discapacitadas o de limpieza, alimentación y compañía, dejan al 
descubierto estas mismas funciones en sus lugares de origen, en donde otras mujeres, abuelas, hermanas o hijas las asumen como pueden.

\subsubsection{LA INFLUENCIA DEL MODELO URBANÍSTICO}

Por una parte, el envejecimiento de la población y mantenimiento de la vida hasta edades muy avanzadas, en muchos casos en situaciones de fuerte dependencia física, exige una mayor dedicación a las personas mayores. En segundo lugar, aunque el número de niños y niñas ha disminuido, la destrucción de espacios públicos para el juego y la transformación de la calle en un lugar agresivo invadido por los coches, obligan a cuidar de una forma mucho más intensiva. La infancia ya no puede estar jugando en las plazas sin vigilancia, ni va sola al colegio hasta edades muy avanzadas.

El crecimiento urbano desbocado juega un papel fundamental en la dificultad que existe en nuestras sociedades para garantizar el bienestar y el cuidado de la vida humana. Del mismo modo que el hipertrofiado entramado de carreteras y el excesivo transporte motorizado fragmentan y deterioran los ecosistemas y envenenan el aire que respiramos, también escinden y alejan los espacios físicos en los que se desarrollan las diferentes dimensiones de la vida de las personas, obligando a invertir una gran cantidad de horas en los desplazamientos del trabajo a casa, al colegio, a la casa de los mayores que hay que atender, al médico, o a la compra.

La separación entre hogar y trabajo fue una contribución al proceso de desarrollo del capitalismo industrial que acentuó las distinciones funcionales y biológicas entre mujeres y hombres. La división de tareas se consolidó como el modo más eficiente, racional y productivo de organizar el trabajo, los negocios y la vida social.

El nuevo modelo de desarrollo debía disponer de una organización territorial y social que permitiese su funcionamiento eficaz. El urbanismo racionalista propuso una ciudad ordenada, limpia y segmentada física y socialmente frente a la ciudad antihigiénica y abirragada que pervivía en el siglo XIX. Esta concepción de ciudad, que separa de forma clara las áreas residenciales, comerciales y productivas, y las redes de transporte, pasa a definir la configuración territorial y urbana durante las primeras décadas del siglo XX en la mayor parte de las ciudades anglosajonas y definirá la ordenación territorial en el resto del mundo hasta nuestros días.

El modelo de ciudad y de progreso es concebido por hombres que no comprenden la importancia del trabajo de cuidados ni la necesidad de realizar varias funciones simultáneamente en el mismo espacio que les caracteriza. Por ello la ordenación de territorio gestada dificulta el mantenimiento de esta actividad esencial y profundiza la desresponsibilización de los hombres como colectivo, poniendo la maquinaria de la edificación y del urbanismo al servicio del sistema económico. Con estas premisas, la ordenación del territorio se convertía en una nueva forma de agresión a las mujeres (Vega, 2004:30). 
Existen importantes trabajos de mujeres urbanistas que proponen una ordenación del territorio más acorde con las necesidades de los cuidados. Un ejemplo es el Colectivo de Mujeres Urbanistas, creado en Madrid en 1995, que se configuró como un grupo de debate y acción social dedicado a trabajar por la equidad de género y generación de nuevas propuestas en el espacio construido. Muchas de las propuestas de las mujeres urbanistas son coincidentes con las que se realizan desde el movimiento ecologista. Aquel modelo de ciudad que se perfila como más adecuado para mantener el bienestar de las personas y garantizar la reproducción social, es también mejor para el conjunto de los ecosistemas urbanos.

En el marco de la crisis actual, la precarización laboral y la amenaza del paro obligan a plegarse a los ritmos y horarios que impone la empresa. La pérdida de redes sociales de apoyo mutuo fuerza a resolver los asuntos cotidianos de una forma mucho más individualizada con las dificultades añadidas que eso supone.

La crisis del sistema de cuidados que hasta el momento garantizaba el mantenimiento de las condiciones básicas de bienestar humano (que recaía fundamentalmente las mujeres) se hace especialmente grave ante el progresivo desmantelamiento y privatización de los servicios sociales que trataban de paliar algunos de estos problemas. La reproducción social se relega al ámbito invisible del hogar en donde son mayoritariamente las mujeres quieres cargan con el peso del ajuste.

\subsection{LA LÓGICA DE LOS BENEFICIOS CONTRA LA LÓGICA DE LA VIDA}

En las sociedades capitalistas aquello que produce beneficio económico es prioritario frente a lo que beneficia a las personas. Y muchas veces ambas cosas no coinciden.

La lógica que subyace al funcionamiento de lo vivo fricciona con la organización de un modelo económico que pretende ser hegemónico, y que se basa en la expansión y crecimiento permanente. La una pretende el mantenimiento de los procesos vitales y puede contribuir a la resolución de las necesidades humanas, mientras que la otra busca la concentración de poder y el beneficio desvinculados de criterios éticos. Existe un planteamiento paralelo si hablamos del mantenimiento del bienestar de las personas en el marco de éste sistema económico. Estamos de acuerdo con Picchio (1992) cuando afirma que existe una honda contradicción entre el proceso de reproducción de personas y el proceso de acumulación de capital. Los objetivos de ambas lógicas y las estrategias para lograrlos no son sólo diferentes, sino que muchas veces son difícilmente conciliables porque obedecen a prioridades muy diferentes.

El hecho llamativo de que los seres humanos vivamos de espaldas a nuestra propia supervivencia tiene que ver con dos elementos articuladores de nuestra cultura: la desvalorización del trabajo de reproducción social que promueve el orden 
social patriarcal y el tratamiento que la cultura occidental y el capitalismo dan a la naturaleza como recurso susceptible de apropiación (Federici, 2010).

La invisibilización de los trabajos sobre los que se asienta la supervivencia y la vida buena son herramientas que el patriarcado y el capitalismo moderno, dos sistemas que actúan de forma sinérgica, usan en su provecho.

El mercado se nos presenta como protagonista de la actividad humana, aunque su aportación a nuestra supervivencia es mucho menor que la que tiene el trabajo asociado a la reproducción social y las producciones de la naturaleza. Para ejemplificar esta desproporción, tanto la economía feminista como la ecológica usan la metáfora del iceberg. Flotando en la superficie visible está el mercado. Debajo, haciéndolo flotar, con un tamaño mucho mayor, el trabajo oculto de los hogares y la aportación de los ciclos naturales y de los minerales de la corteza terrestre.

Según Shiva, las sociedades humanas se mueven dentro del funcionamiento de tres esferas económicas: la economía de la naturaleza, la economía de la supervivencia y la economía de mercado. Ésta última ha crecido exponencialmente a costa de las otras dos, que no han hecho más que disminuir y deteriorarse. El problema es que ellas son la base de la última, pues conforman la economía de la vida.

La economía de mercado se desentiende de las necesidades básicas de la sociedad. Para Carrasco, entre la sostenibilidad de la vida humana y el beneficio económico, las sociedades occidentales han optado por este último. Esto significa que las personas no son el objetivo social prioritario, sino que están al servicio de la producción (Carrasco, 2009:183).

La valorización del cuidado lleva a la economía feminista a acuñar la idea de sostenibilidad de la vida humana (Carrasco, 2009:183) bajo un concepto que representa un proceso histórico complejo, dinámico y multidimensional de satisfacción de necesidades que debe ser continuamente reconstruido, que requiere de recursos materiales pero también de contextos y relaciones de cuidado, proporcionados éstos en gran medida por el trabajo no remunerado realizado en los hogares .

"Un concepto que permite dar cuenta de la profunda relación entre lo económico y lo social, que sitúa a la economía desde una perspectiva diferente, que considera la estrecha interrelación entre las diversas dimensiones de la dependencia y, en definitiva, que plantea como prioridad las condiciones de vida de las personas, mujeres y hombres” (Carrasco, 2009:183).

En nuestra opinión, este concepto se incluye dentro de la idea más amplia de sostenibilidad ecológica y social. De acuerdo con Bosch (2005), entendemos la sostenibilidad:

"Como proceso que no sólo hace referencia a la posibilidad real de que la vida continúe -en términos humanos, sociales y ecológicos-, sino a que dicho proceso 
signifique desarrollar condiciones de vida, estándares de vida o calidad de vida aceptables para toda la población. Sostenibilidad que supone, pues, una relación armónica entre humanidad y naturaleza, y entre humanas y humanos. En consecuencia, será imposible hablar de sostenibilidad si no va acompañada de equidad”

\subsection{DEUDA ECOLÓGICA Y DEUDA DE LOS CUIDADOS}

Al analizar la apropiación de los bienes y servicios de la naturaleza y de los tiempos de trabajo femeninos se pueden establecer aún más paralelismos interesantes entre las perspectivas feministas y ecologistas.

Resulta interesante indagar en el paralelismo entre la crisis ambiental y la crisis de los cuidados. Ambas son resultado de la translimitación, en un caso de los tiempos vitales disponibles para el cuidado, en el otro de los recursos que la tierra puede ofrecer. Ambas exportan sus efectos indeseables a territorios lejanos, en un caso en forma de deuda ecológica y en otro en forma de cadenas globales de cuidados.

La huella ecológica es un indicador que traduce a unidades de superficie lo que un estado o una comunidad consumen y los residuos que genera. Según este indicador, si todos los habitantes del planeta tuviesen el estilo de vida similar a la media de la ciudadanía española, se necesitarían tres planetas para sostener ese nivel de consumo.

La deuda ecológica es la que los países ricos han contraído con los países empobrecidos debido al desigual uso de los recursos y bienes naturales, así como la desigual responsabilidad en el deterioro y destrucción del medio físico.

Paralelamente, cabría hablar de la huella de los cuidados de las mujeres como indicador que evidencia el desigual impacto que tiene la división sexual del trabajo sobre el mantenimiento y calidad de vida humana. La huella de los cuidados es la relación entre el tiempo, el afecto y la energía humana que las personas necesitan para atender a sus necesidades humanas reales (cuidados, seguridad emocional, preparación de los alimentos, tareas asociadas a la reproducción, etc) y las que aportan para garantizar la continuidad de vida humana. El balance de la huella de cuidados sería negativo para la mayor parte de los hombres, pues consumen más energías cuidadoras para sostener su forma de vida que las que aportan.

Siguiendo con el paralelismo, desde el feminismo, podría hablarse de deuda de los cuidados, como la deuda que el patriarcado ha contraído con las mujeres de todo el mundo por el trabajo que realizan gratuitamente.

Esta deuda es esencialmente un elemento de visibilización. Aunque podría analizarse e incluso intentar cuantificarse, la reflexión es compleja, pues no puede valorarse de igual forma la huella de una persona sana que la de una enferma, los tiempos dedicados a tareas agradables o los tiempos dedicados a tareas penosas. 
En cualquier caso, lo que sí permite constatar es que existe un desequilibrio profundo que convierte en injusto y socialmente insostenible el modo de reparto de trabajos de cuidado, como es injusto y socialmente insostenible que el mundo se encuentre polarizado entre núcleos ricos que depredan población, capitales y recursos y extensos territorios que se usan como áreas de apropiación y vertido.

La huella de cuidados y la deuda de cuidados pueden ser, como ya lo son la huella ecológica y la deuda ecológica, elementos de denuncia de un orden social basado en la explotación de las mujeres.

\section{EL CAMINO HACIA LA SOSTENIBILIDAD}

Puesto que no es posible un crecimiento económico indefinido dentro de una biosfera de recursos y sumideros finitos y que los límites ya han sido superados, el camino hacia la sostenibilidad está forzosamente marcado por la disminución de la extracción y la generación de residuos. Los datos de huella ecológica publicados por WWF (2010:17), ponen de manifiesto la superación de los límites de la capacidad de carga del planeta a nivel global por encima de un 30\%, aunque de una manera desigual por parte de los distintos países. El informe advierte de que si las demandas continúan al ritmo de crecimiento actual, se necesitarían el equivalente a dos planetas para el año 2030.

Inevitablemente, la organización de las sociedades, los criterios económicos y los principios de convivencia que se establecen en un mundo translimitado son diferentes a los que se desarrollaron en el pasado para un mundo vacío. La imposibilidad de seguir creciendo materialmente en un planeta con límites, deja como única opción la reducción consciente y radical de la extracción de energía y materiales, así como la fuerte restricción en a generación de residuos, y esto hasta ajustarse a los límites de la biosfera.

Nuestro modelo económico, al amparo del paradigma económico neoclásico, ha sido capaz de generar un enorme desarrollo industrial y abundancia de mercancías, pero lo ha hecho costa de poner en peligro el futuro de la humanidad y de generar situaciones de miseria en gran parte del planeta.

Reducir el tamaño de una esfera económica que ha crecido sobre la extracción de minerales finitos y la generación de residuos crecientes no es una opción que podamos o no escoger. El agotamiento del petróleo y de los minerales, el cambio climático y los desórdenes en los ciclos naturales, van a obligar a ello. La humanidad va a tener que adaptarse en cualquier caso a vivir extrayendo menos de la Tierra, plegándose a lo que su producción cíclica puede dar y generando menos residuos. Esta adaptación puede producirse por la vía de la pelea feroz por el uso de los recursos decrecientes o mediante un proceso de reajuste decidido y anticipado con criterios de equidad. 
El reto es aprender a producir valor y felicidad reduciendo progresivamente la utilización de materia y energía. No hay recetas, pero sí de un conjunto de criterios claros, de caminos posibles para superar muchas de las contradicciones. Implica cambiar la mirada sobre la realidad y desprenderse de un modo de vida incompatible con el planeta. Se trata de buscar nuevas formas de socialización, de organización social y económica que permitan librarse de un modelo de desarrollo que prioriza los beneficios monetarios sobre el mantenimiento de la vida.

Una saludable reducción de las extracciones de la biosfera y situar el bienestar de las personas como objetivos social obliga a plantear un radical cambio de dirección. "Descolonizar el imaginario económico" (Latouche, 2008:147) y cambiar la mirada sobre la realidad, promover una cultura de la suficiencia y la autocontención en lo material, cambiar los patrones de consumo, reducir drásticamente la extracción de materiales y el consumo de energía, apostar por las economías locales y los circuitos cortos de comercialización, restaurar una buena parte de la agricultura campesina, disminuir el transporte y la velocidad, aprender de la sabiduría acumulada en las culturas sostenibles y situar el cuidado de las personas en el centro del interés, son algunas de las líneas directrices del tránsito de la sociedad del crecimiento a otro modelo en el que la vida humana digna que se reconozca como parte de la biosfera.

Salir de la lógica androcéntrica sitúa a la economía hipertrofiada en un plano diferente y obliga a responder a las preguntas que realiza la economía feminista: ¿qué necesidades hay que satisfacer? ¿Cuáles son las producciones necesarias para que se puedan satisfacer? ¿Cuáles son los trabajos socialmente necesarios para ello?

\subsection{UNA PRODUCCIÓN LIGADA AL MANTENIMIENTO DE LA VIDA Y NO A SU DESTRUCCIÓN}

La convicción de que tanto la tierra como el trabajo son sustituibles por capital propició que la economía se centrase sólo en el mundo del valor monetario, olvidándose del mundo físico y material.

Al reducir la consideración de valor a lo monetario, muchas cosas quedan ocultas a los ojos del sistema económico. Suman positivamente el valor mercantil de lo producido, pero no restan los deterioros asociados o la merma de riqueza natural. Al contabilizarse sólo la dimensión creadora de valor económico y vivir ignorantes de los efectos negativos que comporta esa actividad, se alentó el crecimiento de esa "producción” (en realidad extracción y transformación) de forma ilimitada, cifrándose el progreso de la sociedad en el continuo aumento de los "bienes y servicios" obtenidos y consumidos.

Esta forma de razonar sitúa el objetivo de la economía en incrementar las producciones sin que importe la naturaleza de las mismas, celebrándose el crecimiento 
de actividades que son a todas luces dañinas para el conjunto de las personas y el medio ambiente, que crecen a expensas del deterioro los servicios ecosistémicos y de invisibilizar los tiempos de trabajo necesarios para la reproducción social.

La ceguera de los instrumentos económicos ante los motivos reales de la bonanza económica de los últimos años (el crecimiento excesivo del crédito y la burbujas inmobiliaria, la hipertrofia de determinados sectores o la dependencia de la financiación exterior,) pone de manifiesto la necesidad de olvidar indicadores como el PIB para interpretar el éxito económico y adoptar un conjunto de indicadores multicriterio que consideren otras dimensiones como son los flujos físicos, la apropiación de la producción primaria neta o los tiempos necesarios para las tareas de cuidados.

En los mercados capitalistas, la obligación de acumular determina las decisiones que se toman sobre qué se produce, cómo y cuánto se produce, acerca de cómo estructurar los tiempos, los espacios o las instituciones legales.

Desde el punto de vista de la sostenibilidad, la economía debe ser el proceso de satisfacción de las necesidades que permiten el mantenimiento de la vida para todas las personas. Este objetivo no puede compartir la prioridad con el lucro. Si prima la lógica de la acumulación, las personas no son el centro de la economía. El beneficio no se puede conciliar con el desarrollo humano, o es prioritario uno, o lo es el otro y esta opción determina las decisiones que se toman en lo social y en lo económico.

\subsubsection{BIOMÍMESIS, UNA FORMA DE PRODUCIR COMPATIBLE CON LA NATURALEZA}

Riechmann expone que la naturaleza nos proporciona el modelo para una economía sostenible y de alta productividad. La economía de la naturaleza es:

"Cíclica, totalmente renovable y autorreproductiva, sin residuos, y cuya fuente de energía es inagotable en términos humanos: la energía solar en sus diversas manifestaciones (que incluye, por ejemplo, el viento y las olas). En esta economía cíclica natural cada residuo de un proceso se convierte en la materia prima de otro: los ciclos se cierran” (Riechmann, 2005)

Estas son las mejores pautas para reconvertir los procesos productivos hasta hacerlos compatibles con la naturaleza. Cara a favorecer el cierre de ciclos de materiales, Naredo ha planteado que, además de registrarse los costes de la extracción y manejo de los minerales de la corteza terrestre, deben consignarse los costes de reposición, es decir de transformación de los residuos en recursos naturales ya que de los contrario, al no restar en las cuantas la degradación, se favorece el deterioro del patrimonio natural. 
La fiscalidad ecológica, en esta línea pretende cambiar la base de los impuestos desde el valor añadido hacia el flujo material que se produce desde la extracción de recursos al sistema económico y la posterior vuelta de los residuos.

Promover los mercados locales y regionales y la distribución cercana es una necesidad en un mundo con las fuentes energéticas de origen fósil en declive y con una urgente necesidad de reducir emisiones de gases de efecto invernadero.

Además, resulta esencial exigir el principio de precaución, de forma que no se comercialicen o se extiendan tecnologías o productos que no hayan demostrado de una forma convincente que no son nocivas para el medio y para las personas. En la actualidad más bien se imponen las "innovaciones" y se suponen inocuas hasta que se demuestre lo contrario.

\subsection{UN CAMBIO RADICAL EN EL MODELO DE TRABAJO}

En una sociedad que necesariamente tendrá que aprender a vivir bien con menos material, que deberá adoptar modelo de producción y consumo más sobrio y más equitativo, es de capital importancia reflexionar sobre qué trabajos son social y ambientalmente necesarios, y cuáles son aquellos que no es deseable mantener. La pregunta clave para valorarlos es en qué medida facilitan el mantenimiento de la vida en equidad. Se trata de un tema especialmente polémico en un momento en el las personas paradas se cuentan por millones y en el que los gobernantes denominan austeridad al proceso de expolio de lo común que queda y al expolio de los recursos públicos para retomar el crecimiento de los beneficios.

El gran escollo que se suele plantear al habar de transición hacia estilo una vida mucho más austero (ecológicamente hablando) es el del empleo. Históricamente, la destrucción de empleo ha venido en los momentos de recesión económica. Es evidente que un frenazo en el modelo económico actual termina desembocando en el despido de trabajadores y trabajadoras. Sin embargo, algunas actividades deben decrecer y el mantenimiento de los puestos de trabajo no puede ser el único principio a la hora de valorar los cambios necesarios en el tejido productivo. Hay trabajos que no son socialmente deseables, como son la fabricación de armamento, las centrales nucleares, el sector del automóvil o los empleos que se han creado alrededor de las burbujas financiera e inmobiliaria. Las que sí son necesarias son las personas que desempeñan esos trabajos y por tanto, el progresivo desmantelamiento de determinados sectores tendría que ir acompañado por un plan de reestructuración en un marco fuertes coberturas sociales públicas que protejan el bienestar de trabajadores y trabajadoras.

Una red pública de calidad de servicios básicos como son la educación, la sanidad, la atención a personas mayores, enfermas o con diversidad funcional requiere personas. Igualmente las tareas de rehabilitación, de reparación, las que giran en 
torno a las energías renovables o a la agricultura ecológica pueden generar empleo; en general, todas las que tengan que ver con la sostenibilidad, necesitan del esfuerzo humano.

Los trabajos de cuidados, que históricamente han realizado las mujeres, los que sirven para mantener o regenerar el medio natural, los que producen alimentos sin destruir los suelos y envenenar las aguas, así como los que consolidan comunidades integradas en su territorio, facilitan el mantenimiento de la vida en equidad y por ello son trabajos deseables. También lo son los que sirven para detener la destrucción de los territorios.

La mirada desde el prisma de la sostenibilidad nos ofrece un panorama del mundo del trabajo completamente diferente del actual. Si intentáramos clasificar los trabajos en relación con su aportación a la calidad de vida, el orden de valoración social sería justamente el contrario. Irían primero la crianza, la producción de alimentos agroecológica, los trabajos dirigidos a la salud y la higiene,... y en los últimos puestos quedarían seguramente los que realizan los ejecutivos de las bolsas financieras, los fabricantes de armas y los que promueves infraestructuras innecesarias. Podríamos diferenciar con propiedad entre trabajos ligados a la producción de la vida y trabajos que provocan su destrucción.

Se hace imprescindible revisar y transformar profundamente el actual modelo de trabajo. Como hemos comentado, no basta que con que el cuidado se reconozca como algo importante si no se trastoca profundamente el modelo de división sexual del trabajo. Es preciso romper el mito de que las mujeres son felices cuidando. Cuidar es duro y se hace por obligación, porque no se puede dejar de hacer.

El reparto equitativo de las tareas domésticas no sólo permitiría que los hombres se hagan conscientes de la magnitud, importancia, y muchas veces penosidad de estos trabajos, sino que seguramente pondría en marcha un cambio cultural de enorme dimensión: variaciones en los usos de los tiempos de vida, en el aprecio por el mantenimiento y la conservación, en la comunicación, en las formas de vida comunitaria, en la vinculación entre el espacio público y privado, en la consideración de los espacios no monetizados...

En el ámbito de la reproducción social y las tareas domésticas y relacionadas con el cuidado, los servicios públicos son esenciales si queremos que la sociedad en su conjunto se responsabilice de ellos. Dejarlos relegados a la individualidad de cada hogar supone que los hombres sólo se responsabilizarán de ellos en el caso de que la correlación de fuerzas en el ejercicio del poder en cada hogar permita no se resuelva bajo la lógica patriarcal. En muchos hogares, la única posibilidad de que las mujeres no se vean obligadas a realizar estos trabajos en soledad es que existan servicios públicos que los cubran. 
La valoración y remuneración justa de estos trabajos es también importante para que los hombres se incorporen. Si los trabajos relacionados con el cuidado tienen prestigio social y están decentemente remunerados, es casi seguro que los hombres querrán hacerlos.

El cuidado, como exigencia para el mantenimiento de la vida, es un requerimiento de la sostenibilidad y tiene que ser asumido por la sociedad, no es una obligación sólo para las mujeres. La cultura del cuidado tendrá que ser rescatada y servir de base a una sociedad social y ecológicamente sostenible.

La disminución de la jornada laboral y el reparto de todos los tiempos de trabajo necesario (remunerado y doméstico) podrían permitir articular otra sociedad diferente. Ahora, la disminución de los beneficios se repercute directamente sobre los puestos de trabajo asalariados, pero podría repercutir sobre los "bonus” y reparto de dividendos a accionistas o sobre algunos salarios, que muy bien podrían bajar sin poner en peligro la subsistencia de quienes los perciben. Además, es preciso tener en cuenta que existen fórmulas empresariales, como las cooperativas, en las que el objetivo primordial no es maximizar el beneficio, sino el mantenimiento de los puestos de trabajo.

\subsection{IGUALDAD Y DISTRIBUCIÓN DE LA RIQUEZA}

Tradicionalmente, se defiende que la distribución está supeditada al crecimiento de la producción. La economía neoclásica presenta una receta mágica para alcanzar el bienestar: incrementar el tamaño de la "tarta”, es decir, crecer, soslayando así la incómoda cuestión del reparto. Sin embargo, hemos visto que el crecimiento contradice las leyes fundamentales de la naturaleza y que no puede tener más que un carácter transitorio y a costa de generar una gran destrucción. Así, el bienestar vuelve a relacionarse con la cuestión esencialmente política de la distribución.

El reparto de la tierra será en el futuro un asunto nodal. La tarea será sustraer tierra a la agricultura industrial, a la especulación urbanística, a la expansión del asfalto y el cemento y ponerla a disposición de sistemas agroecológicos locales.

La exploración de propuestas como la renta básica de ciudadanía o los sueldos complementarios se hace urgente. Igualmente sería interesante considerar la posibilidad de establecer una renta máxima. Del mismo modo que existen muchos empleos precarios e insuficientemente remunerados, hay personas que podrían disminuir el salario neto sin que se viesen afectadas sus condiciones de vida.

Reducir las desigualdades nos sumerge en el debate sobre la propiedad. Paradójicamente nos encontramos es una sociedad que defiende la igualdad de derechos entre las personas que la componen y que sin embargo asume con toda naturalidad enormes diferencias en los derechos de propiedad. En una cultura de la 
sostenibilidad habría que diferenciar entre la propiedad ligada al uso de la vivienda o el trabajo de la tierra, de aquellas otras ligadas a la acumulación ya sea en forma de bienes inmuebles o productos financieros y poner coto a éstas última, ya que suponen situar fuera del alcance de otras personas la posibilidad de satisfacer necesidades básicas.

Cara a limitar la acumulación y reducir gradientes de desigualdad es fundamental modificar el sistema monetario internacional para establecer regulaciones que limiten la expansión financiera globalizada, regular la dimensión de los bancos, controlar su actividad, aumentar el coeficiente de caja, limitar las posibilidades de creación de dinero financiero y dinero bancario y suprimir los paraísos fiscales de modo que no constituyan vías de escape para que los oligarcas sitúen su patrimonio y negocios fuera de las leyes estatales.

Apostar por la redistribución equitativa de la riqueza supone unos servicios públicos fuertes, una fiscalidad progresiva y que la prioridad del gasto público se oriente al bienestar: sanidad, educación, protección y cuidado de la población.

En definitiva, se trata de cambiar los criterios que hoy prevalecen por otra racionalidad económica que se someta a las exigencias sociales y ambientales que permiten el mantenimiento de la vida. Orientar las decisiones económicas hacia la igualdad no es sólo cuestión de normativa o instrumentos económicos, sino de impulsar también cambios culturales en dirección contraria de los que se han venido estimulando en las últimas décadas.

\subsection{TEJER ALIANZAS: CONSTRUIR MAYORÍAS}

En el momento actual, dentro de los movimientos sociales y políticos que defienden la necesidad de una transformación que conduzcan a la sostenibilidad ecológica y humana y a la justicia social, la potencia del análisis crítico de la realidad y las propuesta de cambio no guardan relación con las escasas fuerzas que existen para forzar estos cambios.

Aunque cada vez son más las iniciativas y movimientos de todo tipo que comparten análisis y cuyas propuestas son convergentes y no excluyentes, aún se está lejos de confluir y articular una base sólida que exija y apoye los cambios necesarios. Las medidas y políticas con las que se pretende salir de la crisis son justamente las contrarias a las necesarias desde el punto de vista del bienestar de las personas.

Si queremos forzar cambios, habrá que dar la batalla en el ámbito de las ideas, y en la práctica económica, ecológica, social y política. Pero sobre todo será necesario construir poder colectivo y sumar mayorías que puedan impulsar y exigir un cambio. 
No cabe pensar que el colapso social y ambiental venga en nuestra ayuda. Si no somos capaces de articular movimiento, lo que venga detrás de este capitalismo puede ser aún peor. Por ello tendremos que superar viejas tendencias en la forma de militar o de ser activista que han hecho de cada diferencia un motivo de fragmentación, que han convertido en enemigo a aquel del que menos nos separaba.

Desarrollar la crítica feroz es fácil, estamos muy acostumbrados a ello. Es más difícil buscar acercamientos, convivir con algunas diferencias, acostumbrarnos a hacer trechos de camino en una dirección que nos convenga sin romper demasiado pronto porque la meta a la que queremos llegar no es exactamente igual.

Los seres humanos evolucionaron gracias a la cooperación y el apoyo mutuo y nosotros seguimos siendo seres humanos que sólo conseguiremos fuerza para imponer cambios a partir de la construcción colectiva, de la búsqueda incansable de acuerdos y del cuidado a lo único que tenemos para dar la batalla: nuestros compañeros y compañeras.

\section{BIBLIOGRAFÍA}

BALLENILLA, M. y BALLENILLA, F. (2007) La Tasa de Retorno Energético. Madrid. El Ecologista, ${ }^{\circ} 55$.

BOSCH, A., CARRASCO, C. y GRAU, E. (2005). Verde que te quiero violeta. Encuentros y desencuentros entre feminismo y ecologismo. En E. Tello, "La historia cuenta”, Madrid, Ediciones El Viejo Topo

CARRASCO, C. (2009) Tiempos y trabajo desde la experiencia femenina Papeles de Relaciones Ecosociales y Cambio Global. No 108

DUARTE, C. (coord) (2006) Cambio Global. Impactos de la actividad humana sobre el sistema Tierra. Madrid. CSIC

FEDERECI, S. (2010) Calibán y la bruja. Mujeres, cuerpo y acumulación originaria. Madrid. Traficantes de Sueños

FERNÁNDEZ DURÁN, R. (2010) El Antropoceno: la crisis ecológica se hace mundial. La expansión del capitalismo global choca con la Biosfera. Madrid. Coeditores. Virus y Libros en Acción

GARCÍA, E. (2005) El Cambio más allá de los límites al crecimiento: un nuevo referente para el realismo en la sociología ecológica. Universidad de Valencia.

GARCÍA, E. (2004) Medio ambiente, y sociedad: la civilización y los límites del planeta. Editorial Alianza Ensayo

HUBBERT, K. Energy from Fossil Fuels en Science vol 199. www.eoearth.org 1949

LATOUCHE, S. (2008), La apuesta por el decrecimiento Barcelona Icaria

MARTÍNEZ ALIER, J. (2004), El ecologismo de los pobres: conflictos ambientales y lenguajes de valoración. Icaria

MEADOWS, D. H. (1972), Los límites al crecimiento: informe al Club de Roma sobre el predicamento de la humanidad, México D.F. Fondo de Cultura Económica

MEADOWS, D., RANDERS, J. y MEADOWS, D. (2004), Limits to growth: the 30 years update, White River Junction (UT) Chelsea: Green 
MORENO, JM (coord) (2005) Principales conclusiones de la evaluación preliminar de los impactos en España por efecto del cambio climático. Ministerio de Medio Ambiente

NAREDO J.M. (2006) Raíces económicas del deterioro ecológico y social. Más allá de los dogmas. Siglo XXI. Madrid

OBERHUBER, T. (2004) Camino de la sexta gran extinción en Ecologista , n.41. Ecologistas en Acción.

PICCHIO, A. (1992) Social Reproduction: the political economy of Labour Market, Cambridge University Press

PROGRAMA DE NACIONES UNIDAS PARA EL DESARROLLO EN MÉXICO (2008) Guía de Recursos de género para el cambio climático. México, PNUD

RIECHMANN, J. (2005) Biomímesis. Ensayos sobre imitación de la naturaleza, ecosocialismo y

autocontención, Madrid, La Catarata

VEGA, P. (2004) Las mujeres y el deterioro ecológico provocado por el actual modelo territorial. ¿Cómplices o víctimas?, Madrid, “El Ecologista”, No 39

WWF (2010) Planeta Vivo. Informe 2010. Biodiversidad, biocapacidad y desarrollo, Madrid, WWF España 